
\title{
Reseracth Sulure \\ Under Age Five Children Survival Times in Nigeria: A Bayesian Spatial Modelling Approach
}

Osafu Augustine Egbon

University of São Paulo

Mariella Ananias Bogoni

Universidade Federal de São Carlos

Bayowa Teniola Babalola ( $\sim$ bayowa.babalola@kiu.ac.ug)

Kampala International University

Francisco Louzada

University of São Paulo

\section{Research Article}

Keywords: Hazard model, Bayesian hierarchical model, Under-five mortality, survival probability

Posted Date: November 12th, 2021

DOI: https://doi.org/10.21203/rs.3.rs-1042906/v1

License: (c) (1) This work is licensed under a Creative Commons Attribution 4.0 International License.

Read Full License 


\title{
RESEARCH
}

\section{Under Age Five Children Survival Times in Nigeria: A Bayesian Spatial Modelling Approach}

\author{
Osafu Augustine Egbon ${ }^{1,2}$, Mariella Ananias Bogoni ${ }^{1,2}$, Bayowa Teniola Babalola ${ }^{3 *}$ and Francisco \\ Louzada $^{1}$ \\ ${ }^{1}$ Institute of Mathematical and Computer Sciences, University of São Paulo, São Carlos, Brazil. \\ 2 Department of Statistics, Universidade Federal de São Carlos, São Carlos, Brazil. \\ ${ }^{3}$ Department of Mathematics and Statistics, Kampala International University, Kampala, Uganda.
}

\footnotetext{
${ }^{*}$ Correspondence:

bayowa.babalola@kiu.ac.ug

${ }^{3}$ Department of Mathematics and

Statistics, Kampala International

University, Kampala, Uganda

Full list of author information is

available at the end of the article
}

Abstract
Background: Nigeria is among the top five countries in the world with the highest under-five mortality rates. In addition to the general leading causes of under-five mortality, studies have shown that disparities in sociocultural values and practices across ethnic groups in Nigeria influence child survival, thus there is a need for scientific validation.

Methods: The approach commonly adopted in multilevel modeling of the under-five mortality rate in Nigeria is to consider child death status as a binary outcome variable. In this manner, information about the time localization of mortality risk is lost in the process. This study quantified the impact of socioeconomic and demographic factors, proximate and biological determinants, and environmental factors on the risk of under-five mortality in Nigeria using a Bayesian hierarchical hazard model with time to death outcomes. The Exponential, Gamma, Log-normal, Weibull, and Cox hazard models in a mixed effect framework with spatial component were considered, and the best model was selected for inference. In addition, the Kaplan Meier survival curve, Nelson Aalen hazard curve, and components survival probabilities were estimated.

Results: Findings show that gender, maternal education, household wealth status, source of water and toilet facility, residence, mass media, frequency of antenatal and postnatal visits, marital status, place of delivery, multiple births, who decide healthcare use, use of bednet are significant risk factors of child mortality in Nigeria. The mortality risk is high among the maternal age group below 24 and above 44years, and birth weight below $2.5 \mathrm{Kg}$ and above $4.5 \mathrm{Kg}$. The under-five mortality risk is severe in Kebbi, Kaduna, Jigawa, Adamawa, Gombe, Kanu, Kogi, Nasarawa, Plateau, Sokoto states in Nigeria.

Conclusions: This study accentuates the need for special attention for the first two months after childbirth as it is the age group with the highest expected mortality. A practicable way to minimize death in the early life of children is to improve maternal health care service, promote maternal education, encourage delivery in healthcare facilities, positive parental attitude to support multiple births, poverty alleviation programs for the less privileged, and a prioritized intervention to Northern Nigeria.

Keywords: Hazard model, Bayesian hierarchical model, Under-five mortality, survival probability. 


\section{Background}

The under-five mortality rate is still a persistent problem in the world. According to [1], the global number of under-five deaths was estimated as $5.2(5.0,5.6)$ million in 2019, which implies that approximately 14,000 children died every day before attaining their fifth birthday in 2019. Since 1990, the global under-five mortality rate has declined by 59\%, fallen from 93 deaths per 1000 live births in 1990 to 38 deaths per 1000 live births in 2019, [2]. Although there is a substantial global reduction in under-five mortality rates since 1990, disparities exist across countries. Sub-Saharan Africa region remains the highest-burden region of under-five mortality in the world, with one in thirteen children dying before reaching the fifth birthday thus, placing the region at top risk region of under-five deaths, [2]. According to WHO, half of the global under-five deaths occurred in five countries, including Nigeria.

Nigeria is one of the countries that presented a high under-five mortality rate of 117 per 1, 000 live births in 2019, [3]. The country is listed among the top five countries with the highest under-five mortality rate in 2019. A collective effort of the government, stakeholders and non-governmental organizations in the fight against under-five mortality to improve the well-being of children has led to a consistent declining in the early child mortality rate in Nigeria, [3]. Despite several interventions to lower the under-five mortality index in Nigeria, the rate is still relatively high. The slow declining pace of the rate may hinder the attainment of the Sustainable Development Goal (SDG) to end preventable under-five death in 2030. The SDG target is to reduce under-five mortality to at least 25 per 1,000 live birth by 2030, [2]. Given the statistics, there must be at least a $50 \%$ decline in early childhood mortality in Nigeria to attain the SDG by 2030, [4]. Therefore, the under-five mortality burden in Nigeria calls for rapid attention to scale down and below the global mortality average rate.

Leading causes of under-five death include preterm birth complications, birth trauma, pneumonia, congenital anomalies, diarrhea, and malaria, [2], which are preventable with affordable interventions. However, under-five death in Nigeria has been attributed to additional factors including, disparities in sociocultural values and practices, [5]. Cultural believes either positively or negatively influence the health status of children, and consequently introduced heterogeneity in improving the survival and well-being of children. For instance, the disparity of persisting low birth in health facilities, low number of births attended by healthcare service providers, low number of antenatal and postnatal care utilization among different ethnic groups in the country, $[6,7,8]$. Nonetheless, there are enormous geographical variations of the under-five mortality rate in the country. According to [4] report, most under-five deaths in Nigeria occurred in the Northern states. Thus, there is a need for scientific validation of the contribution of socioeconomic and demographic factors increasing the risk of child mortality in Nigeria.

Several types of research have been conducted to contribute to the fight against child mortality in Nigeria. For instance, [5] examined the effect of ethnicity on under-five mortality in children and found that the risk of mortality significantly varies between ethnic groups in Nigeria. It was found that the mortality rate is high among Hausa, Fulani, and Kanuri tribes in Nigeria. [9] investigated the effect of child characteristics on under-five mortality in Nigeria. They found that birth 
between maternal ages of 20-24 has a lower risk of mortality. [10] determined the socioeconomic indicators contributing to child mortality in Nigeria, and surprisingly, concluded that attendance of skilled health workers during delivery was associated with an increased risk of neonatal death. [11] examined the Spatio-temporal distribution of under-five mortality rate in Nigeria and found that there is spatial clustering and geographical disparities between states in Nigeria. Recently, [12] examined the effect of spatial distribution and other covariates on under-five mortality deaths in Nigeria.

Many researchers adopted the indirect method of estimating mortality rates in Nigeria, where the survival status of children to a cohort is used to estimate the mortality rate. The implicit assumption of this approach is that the births of a cohort are the number of children born in a period, [13]. This assumption is hardly satisfied as studies have shown a disparity in a maternal age group to the risk of under-five mortality, [14]. Moreover, an approach commonly adopted among these researches in multilevel modeling of under-five mortality rate is to consider the death status of a child as a binary outcome variable, [12]. In this manner, information about the time localization of mortality risk is lost in the process. Thus, give rise to a poor time resolution of under-age five mortality and could lead to bias estimates of the variance, $[15,16]$.

This study aims to quantify the impact of socioeconomic and demographic factors, proximate and biological determinants, and environmental factors on the risk of under-five mortality in Nigeria. This study adopted the direct estimation method of mortality rates, where the date of birth, survival status, and ages at death of children was utilized for the analysis. In this manner, the heterogeneity in the mortality risk between age 0 to 59 months is incorporated for inference. For instance, a heap of under-five deaths a few months after birth can be incorporated into the modeling framework for estimating the risk at these age groups. Moreover, this study accounted for the uncertainty in the geographical heterogeneity of under-five mortality rates between the states in Nigeria.

This study adopted the Bayesian hierarchical hazard modeling framework to formulate a Gaussian latent model, which comes with computational benefits. The covariates describing the characteristics of children were incorporated into the hazard function through a mixed effect linear predictor. Five parametric hazard models were considered which include, Exponential, Gamma, Log-normal, Weibull, and Cox hazard models. These models were considered given that we do not have certainty of the model that best explains the event chain in the data. The Deviance Information criterion (DIC) and Watanabe-Akaike Information Criterion (WAIC) were used to check for model adequacy. The most appropriate model was used for inferences. Moreover, non-parametric methods such as Kaplan-Meier, Nelson-Aalen, and Demographic and Health survey methodology were adopted for the estimation of

the overall survival probabilities, cumulative hazard rates, and component survival probabilities, [13].

\section{Materials and Method}

\section{Study Area and Data Collection}

The data used in this study were pooled from the 2018 Nigeria Demographic and Health Survey (NDHS), implemented by the National Population Commis- 
sion (NPC). The survey took place between 14th August and 29th December 2018. The sampling frame adopted is the population and Housing Census conducted by the NPC in 2006. The Enumeration Areas (EA) in the 2006 Census served as the survey primary sampling units. The survey implemented a two-stage stratification sampling technique to include EAs in the selection. In the first stage, samples of 1400 EAs were drawn with probability proportionate to size. The resulting listing of households served as the sampling frame in the second stage. A total of 40,427 women were interviewed successfully, yielding a response rate of $99 \%$. Children born within the last 59 months to the interview day were eligible to be included in the study. The age at death of children and the present age of children alive at the interview day was of prime interest. This analysis included a record of 33, 924 children after data piping.

According to the World Health Organization classification, the toilet facility and source of water supply were classified as either improved or unimproved. The improved toilet facility includes flush or pour-flush to a piped sewer system, septic tank or pit latrine, ventilated improved pit latrine, pit latrine with slab, and composting toilet. Similarly, the improved water source includes piped household water connection inside the users dwelling, public taps or standpipes, tube wells or boreholes, protected dug wells, protected springs and rainwater collection, [17].

The survival times for dead children $t_{d}$ and for those who were alive at the time of interview $t_{a}$ were computed as $t_{d}=$ Age at death (in years) $/ 12$ and $t_{a}=$ [Date of interview - Date of birth (in years)]/12.

\section{Statistical Method}

Let $C_{i j}$ be a random variable representing the number of days between the birthday of child $j$ in state $i$ and the day of interview. Let $t_{i j}$ be the number of days a child lived until the event of interest occurred. Define $y_{i j}=\min \left(t_{i j}, C_{i j}\right)$, and $\delta_{i j} \in\{0,1\}$ be a censoring indicator that takes the value 1 if $t_{i j} \leq C_{i j}$ (i.e child $j$ in state $i$ experienced the event of interest (death) and takes the value 0 otherwise. Let $Y$ be a random variable that takes the value $y_{i j}$ for $j=1,2, \ldots, n_{i}$, and $i=1, \ldots, 37$ with density function $f\left(y_{i j} \mid \lambda\right)$. The survival probability is given as

$$
S\left(y_{i j} \mid \boldsymbol{\lambda}\right)=\int_{y_{i j}}^{\infty} f(x \mid \boldsymbol{\lambda}) d x
$$

which is equivalent to $S\left(y_{i j} \mid \boldsymbol{\lambda}\right)=\frac{f\left(y_{i j} \mid \boldsymbol{\lambda}\right)}{h\left(y_{i j} \mid \boldsymbol{\lambda}\right)}$, where $h$ is the hazard function of $Y$. Thus the likelihood function to incorporate the incomplete information is given as

$$
L(Y \mid \boldsymbol{\lambda})=\prod_{i=1}^{37} \prod_{j=1}^{n_{i}} f\left(y_{i j} \mid \boldsymbol{\lambda}\right)^{\delta} S\left(y_{i j} \mid \boldsymbol{\lambda}\right)^{1-\delta}=\prod_{i=1}^{37} \prod_{j=1}^{n_{i}} f\left(y_{i j} \mid \boldsymbol{\lambda}\right)^{\delta}\left(\int_{y_{i j}}^{\infty} f(x \mid \boldsymbol{\lambda}) d x\right)^{1-\delta}
$$

Let $\eta$ be a linear predictor, which is a function of demographic and socio-economic variables, and represent $\eta=g(\lambda)$, where $g$ is a known link function usually determined by the density function of $T$. The model is specified through the hazard function of $T$. Consequently, the likelihood function is written as $L\left(Y \mid g^{-1}(\eta)\right)$. In 
the case of a two parameters model with location and scale parameters $\lambda_{1}$, and $\lambda_{2}$ respectively, $\eta=g\left(\lambda_{1}\right)$ and the likelihood is given as $L\left(Y \mid g^{-1}(\eta), \lambda_{2}\right)$.

In this study, four probability density functions were considered for $Y$, namely, the Exponential, Gamma, Weibull, and Log-normal probability density functions. In addition, the Cox proportional hazard model was also adopted. The summary of the five competing models and their properties for a single child is presented in Table 1.

In the Cox proportional hazard model [18], the hazard rate is defined as

$$
h\left(y_{i j}\right)=h_{0}\left(y_{i j}\right) \exp \left(\eta_{i j}\right)
$$

where $h_{0}($.$) is baseline hazard function. For a given partition of time t$ of death into $K$, we assume $h_{0}$ to be constant over each time interval. That is

$$
h_{0}(t)=\exp \left(c_{k}\right), t \in\left(s_{k}-1, s_{k}\right], k=1,2, \ldots, K .
$$

The parameters $c_{k}, k=1,2, \ldots, K$ are unknown and estimated from the data. The prior model assigned to $c_{k}$ is discussed later in this section.

Table 1: Competing parametric models. $\Lambda$ is the parameterized Gamma density,

\begin{tabular}{|c|c|c|c|c|}
\hline Model & Parameterized Density & Hazard Function & Link & Restriction \\
\hline Exponential & $\lambda \exp (-\lambda y)$ & $\lambda$ & $\eta=\log (\lambda)$ & $\lambda>0$ \\
\hline Gamma & $\frac{1}{\Gamma(s \phi)}\left(\frac{(s \phi)}{\mu}\right)^{(s \phi)} y^{(s \phi)-1} \exp \left(-\frac{(s \phi)}{\mu} y\right)$ & $\frac{\Lambda}{1-\frac{1}{\Gamma(s \phi)} \gamma(s \phi,(s \phi / \mu) y)}$ & $\eta=\log (\mu)$ & $s, \phi>0$ \\
\hline Weibull & $\alpha y^{\alpha-1} \lambda \exp \left(-\lambda y^{\alpha}\right)$ & $\alpha y^{\alpha-1} \lambda$ & $\eta=\log (\lambda)$ & $\alpha, \lambda>0$ \\
\hline Log-normal & $\frac{1}{\tau} \sqrt{\tau} \exp \left(-\frac{1}{2} \tau(\log y-\mu)^{2}\right)$ & $\frac{\sqrt{\tau}}{\sqrt{(2 \pi)}} \exp \left(-\frac{1}{2} \tau(\log y-\mu)^{2}\right.$ & $\eta=\mu$ & $\tau>0$ \\
\hline Cox & $2 .\left(\begin{array}{lll}0 \\
2\end{array}\right.$ & $\begin{array}{c}1-\psi(\sqrt{\tau}(\log y-\mu)) \\
h_{0}(y) \exp (\mu)\end{array}$ & & \\
\hline
\end{tabular}
and $\gamma(.,$.$) is the lower incomplete gamma function.$

The main interest of this study is to determine the risk factors and spatial prevalence of child mortality among under-age five children in Nigeria. Thus, we adopted a Bayesian hierarchical modeling framework. Given the structural additive linear predictor,

$$
\eta_{i j}=\mathbf{x}_{i j}^{T} \boldsymbol{\beta}+\mathbf{z}_{i j}^{T} \boldsymbol{\psi}+\mathbf{w}_{i j}^{T} \boldsymbol{\theta}
$$

where $\boldsymbol{\beta}$ is a $p \times 1$ vector of linear effects, such as mother's education level, household wealth index, place of settlement, e.t.c. $\mathbf{x}$ is a $p \times 1$ design vector for the linear covariates. $\psi$ is a $r \times 1$ vector of non-linear effects to account for variables that varies non-linearly with the time of death, such as mother's age. $\mathbf{z}$ is a $r \times 1$ design binary vector that links the corresponding effect parameters to the time of death. Finally, $\boldsymbol{\theta}$ is a $(37 q) \times 1$ vector of spatial effects to account for heterogeneity between states in Nigeria, and $\mathbf{w}$ is a $(37 q) \times 1$ vector of covariates to incorporate spatial intercepts and slopes, and $q-1$ is the number of spatially varying covariate included in the framework.

In Bayesian framework, the parameters are considered random, that is, the uncertainty in the parameters are represented by a probability distribution. A noninformative multivariate normal distribution was assigned for $\boldsymbol{\beta}$, that is, $\boldsymbol{\beta} \sim$ 
$N_{p}(\mathbf{0}, 100 \mathbf{I})$, where $\mathbf{I}$ is a $p \times p$ identity matrix. Since the mothers' age is equally spaced, a random walk of order two was assigned for the non-linear effects. Let $\boldsymbol{\psi}=\left(\psi_{1}, \psi_{2}, \ldots, \psi_{r}\right)^{T}$, defined

$$
\Delta_{v}=\psi_{v}-2 \psi_{v+1}+\psi_{v+2} \sim N\left(0, \tau_{\psi}^{-2}\right), v=1,2, \ldots, r .
$$

Thus, the joint prior distribution, given by [19] is written as

$$
\pi\left(\boldsymbol{\psi} \mid \tau_{\psi}\right) \propto\left(\tau_{\psi}\right)^{(n-2) / 2} \exp \left\{-\frac{\tau_{\psi}}{2} \sum_{v=1}^{r-2}\left(\Delta_{v}\right)^{2}\right\} .
$$

The set of mothers' ages, $R$, was ordered at regular intervals, that is, $R=$ $\{15,16, \ldots, 49\}$. To facilitate estimation, $\mathrm{R}$ was re-coded into $R=\{1,2, \ldots, 35\}$, and $r=35$. Due to rank deficiency, a sum to zero constraint is employed. The random walk two is flexible, invariant under the addition of linear term to $\boldsymbol{\psi}$, and computationally flexible as it exhibits Markovian property [19].

To account for spatial effects, the Intrinsic Conditional Autoregressive model was adopted. Let $q=1$ and $\boldsymbol{\theta}=\left(\theta_{1}, \theta_{2}, \ldots, \theta_{37}\right)$, that is, $\boldsymbol{\theta}$ account for the spatial intercept and heterogeneity between the states. Let $\boldsymbol{\theta}$ be represented by a Gaussian random field with respect to the Graph $\mathcal{G}=(\mathcal{V}, \mathcal{E})$, where $\mathcal{V}$ is a set of vertices representing the 37 states and $\mathcal{E}$ is the set of edges connecting the states, thus, for $i \neq i^{*},\left\{i, i^{*}\right\} \in \mathcal{E}$ if and only if state $i$ and $i^{*}$ are neighbors. Let $W$ be a $37 \times 37$ adjacency matrix such that $w_{i i^{*}}=1$ if $\{i, j\} \in \mathcal{E}$ and zero otherwise. Let $D$ be a diagonal matrix, where each diagonal entry $d_{i i}$ equals the total numbers of neighbors of state $i \in \mathcal{V}$. Therefore, the precision matrix $\boldsymbol{\theta}$ is given as

$$
Q=\tau_{\theta} \mathbf{D}(\mathbf{I}-\mathbf{W})
$$

Notice that by construction, $Q$ is sparse, and hence, $\boldsymbol{\theta}$ is a Gaussian Markov random field with respect to $\mathcal{G}$. That is, $\theta_{i}$ and $\theta_{i^{*}}$ are conditionally independent given $\boldsymbol{\theta}_{-i i^{*}}$ if and only if $\left\{i, i^{*}\right\} \notin \mathcal{E}$. Therefore, the prior probability density function is given as

$$
\pi\left(\boldsymbol{\theta} \mid \tau_{\theta}\right) \propto \log (|Q|)\left(-\frac{1}{2} \boldsymbol{\theta}^{T} Q \boldsymbol{\theta}\right) .
$$

For the baseline hazard function (4), a first order random walk was assigned for $c_{k}, k=1,2, \ldots, K$. The definition is analogous to Equation (6). Hence,

$$
\begin{aligned}
\Delta_{k} & =c_{k}-c_{k-1} \sim N\left(0, \tau_{c}^{-2}\right) \\
\pi\left(\boldsymbol{\psi} \mid \tau_{c}\right) & \propto\left(\tau_{\psi}\right)^{(n-1) / 2} \exp \left\{-\frac{\tau_{c}}{2} \sum_{k=1}^{r-1}\left(\Delta_{k}\right)^{2}\right\} .
\end{aligned}
$$

A $\log$ gamma hyperprior was assigned to $\log \tau, \log \tau_{c}, \log \tau_{\psi}, \log \tau_{\theta}$, with hyperparameters $\left(1,5 e^{-5}\right)$. A $\log$ gamma prior model was assigned to $\log \phi$ with parameter $(1,0.01)$, and $\alpha$ was assigned a PC-prior distribution given as

$$
\pi(\alpha)=\frac{\lambda}{2} \exp (-\lambda d(\alpha))\left|\frac{\partial d(\alpha)}{\partial \alpha}\right|,
$$


where $d(\alpha)=\sqrt{2 K L D(\alpha)}$, and $K L D(\alpha)=(\Gamma((1+\alpha) / 2) \alpha+\alpha \log (\alpha)-\alpha \gamma+\gamma-\alpha) / \alpha$, $\gamma$ is the Euler's constant, and $\lambda=5$.

To estimate the parameters of interest, the structural additive linear predictor, (Equation 5), is used to formulate a latent variable $\mathcal{X}=\{\boldsymbol{\beta}, \boldsymbol{\psi}, \boldsymbol{\theta}\}$ having a multivariate Gaussian distribution with a sparse precision matrix $\mathbf{Q}\left(\phi_{1}\right)$. Let $\phi_{2}$ be a vector of all the hyperparameters of the likelihood function and the prior distributions, and $\phi=\left(\phi_{1}, \phi_{2}\right)$ with joint distribution $\pi(\phi)$. Thus, the joint posterior distribution is given as

$$
\pi(\mathcal{X}, \boldsymbol{\phi} \mid \mathbf{y}) \propto \pi(\boldsymbol{\phi}) \pi(\mathcal{X} \mid \boldsymbol{\phi}) \prod_{i=1}^{37} \prod_{j=1}^{n_{i}} N\left(y_{i j} \mid \mathcal{X}, \boldsymbol{\phi}\right)
$$

The interest is to make inference from the posterior marginal distributions $\pi(\mathcal{X} \mid \mathbf{y})$ and $\pi(\phi \mid \mathbf{y})$. The formulation (Equation 12) can be estimated using Integrated Nested Laplace Approximation (INLA) described in [20]. The parameters of interest were estimated using the Integrated Nested Laplace Approximation (R-INLA) in $\mathrm{R}$ [21]. R-INLA estimates the posterior marginal distribution of the parameters of interest for inference. The estimation procedure are fully discussed in $[22,23,24,25,26]$. Given that a sum to zero constraints was imposed on the spatial effect, the probability of excess risk was computed from the posterior marginal distribution of the spatial effect, which is given as

$$
\pi\left(\theta_{i}<0 \mid \mathbf{y}\right)=\int_{-\infty}^{0} \pi\left(\theta_{i} \mid \mathbf{y}\right) d \theta_{i}, i=1,2, \ldots, 37
$$

where $\pi\left(\theta_{i} \mid \mathbf{y}\right)$ is the posterior marginal distribution of the $i$ th spatial effect. The interpretation of $\pi\left(\theta_{i} \mid \mathbf{y}\right)$ vary between the models and the link functions adopted in the model specification. For the log-normal and gamma model, a higher probability, $\pi\left(\theta_{i} \mid \mathbf{y}\right)$, indicates a lower risk of death or the higher probability a child residing instate $i$ to survive past age five. Whereas, for Cox proportional hazard, exponential, and Weibull model, a lower value of $\pi\left(\theta_{i} \mid \mathbf{y}\right)$ indicates a lower risk of death or the higher probability a child residing in the state $i$ to survive past age five. Moreover, the integral in Equation 13 was approximated using Monte Carlo samples from the marginal posterior distribution. That is

$$
\pi\left(\theta_{i}<0 \mid \mathbf{y}\right) \approx \frac{\sum_{l=1}^{N} I\left(\theta_{i l}<0\right)}{N}
$$

where $\theta_{i l}$ is the $l$ th sample from $\pi\left(\theta_{i} \mid \mathbf{y}\right), I($.$) is an indicator function and N=$ 10,000 .

Given $n=\sum_{i=1}^{37} n_{i}=33,924$ the number of children under study and with $k \leq n$ distinct time of deaths between birth and time of survey, such that $t_{1} \leq t_{2} \leq \ldots \leq t_{k}$. Note that there is a possibility of having more than one death at each event time. Denote $d_{i}$ as the number of deaths at time $t_{i}$, and $q_{i}$ as the number of children at risk at time $t_{i}$. Thus the Kaplan-Meier estimator of the survival function $S(t)$, and 
the Nelson-Aalen estimator of the cumulative Hazard function $H(t)$ are given as

$$
\hat{S(t)}=\prod_{t_{i}: t_{i} \leq t}\left(1-\frac{d_{i}}{q_{i}}\right), \hat{H(t)}=\sum_{i: t_{i}<t}\left(\frac{d_{i}}{q_{i}}\right) .
$$

The $100(1-\alpha) \%$ confidence interval of $S(t)$ is given as $\hat{S(t)} \pm z(\alpha / 2) \sqrt{V(\hat{S(t))}}$, where

$$
V(\hat{S(t)})=(\hat{S(t)})^{2} \sum_{i: t_{i}<t} \frac{d_{i}}{q_{i}\left(q_{i}-d_{i}\right)} .
$$

For the description of the computational steps for DHS component survival probabilities see $[13,27]$.

\section{Results}

Among the 33,924 children included in the analysis, the estimated censoring rate is $90 \%$. It indicates that for every 1000 live birth, 100 children never attained their fifth birthday. Figure 1(a) presents the overall Kaplan-Meier survival probability estimates without taking any covariate effects into account. The middle black line indicates the mean survival probability, while the red lines indicate the $95 \%$ confidence interval of the survival probability. The curve shows a sharp fall in the survival probabilities among children within the first two months, and further consistently drops with a constant rate until the age of 25 th months. It slowly descends further until a child attains 40 months, and then levels off at a probability of 0.88 . In Figure 1(b), the gender effect was taking into account. Though the survival curves for both males and females relatively have a similar pattern, the survival probability of male children is lower compared with the survival probability of female children. While the survival curve for females level off at 0.906, the curve for male children leveled off at 0.894 . The result shows that the probability of a child surviving past the first two months declines significantly, and consequently, the probability of dying within this period increases with a magnitude of at least $1-0.96=0.04$. In addition, the result also indicates that male children were more likely to die compared with female children, in the same age group, before attaining their fifth birthday. Figure 2a presents the cumulative hazard rate computed using the Nelson Aalen estimator of cumulative hazard, and Figure $2 \mathrm{~b}$ presents the cumulative component probabilities of a child dying before reaching 59 months at each age group. In Figure 2a, there is a sharp increase in the cumulative hazard rates within the first two months after birth. The curve continued growing at a constant rate until 25 months, where the growth rate declined and then leveled off at 45 . In Figure $2 b$, the highest component probability jump between age groups occurred at 0 months. It is followed by a jump from the age group $3-5$ months to $12-23$ months and $12-23$ months to $24-26$ months.

The results of the adopted nonparametric methods are consistent, and it indicates a higher risk of under-five mortality within the first two months after birth and it should be considered as a hot period in time localization. Improving the well-being of children at this stage could significantly scale down the child mortality index in Nigeria. 


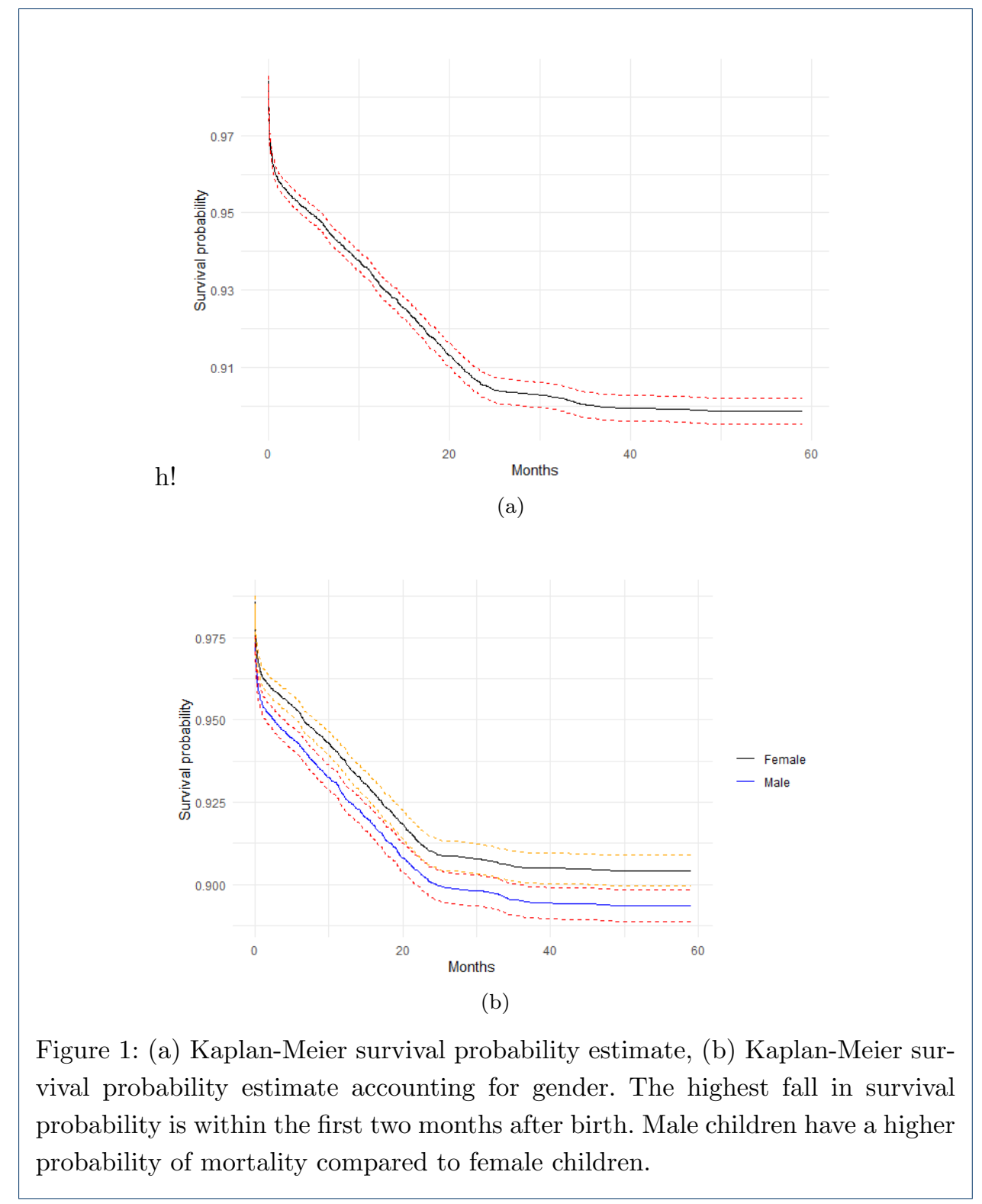

To account for the demographic and socioeconomic key determining factors of under-five mortality, five hazard models, including Exponential, Gamma, Weibull, Log-normal, and Cox hazard models were adjusted. In addition, these models were with and without the inclusion of spatial components. To examine model adequacy, the Deviance Information Criterion (DIC) and the Watanabe Akaike information (WAIC) were estimated. A model is said to be better definitely if the DIC is at least ten units lower than that of a competing model, and on the contrary, there is no fixed threshold for lower WAIC, [28]. Consequently, from Table 2, the log-normal model, with spatial effect, is the most appropriate model for the analysis, and thus, only the results of the log-normal model are presented. However, the presented result is consistent for all the fitted models. It is important to mention that the interpretation of the models' effects varies between the models considered and depends on the link function adopted. For the log-normal model and the link function considered in this 


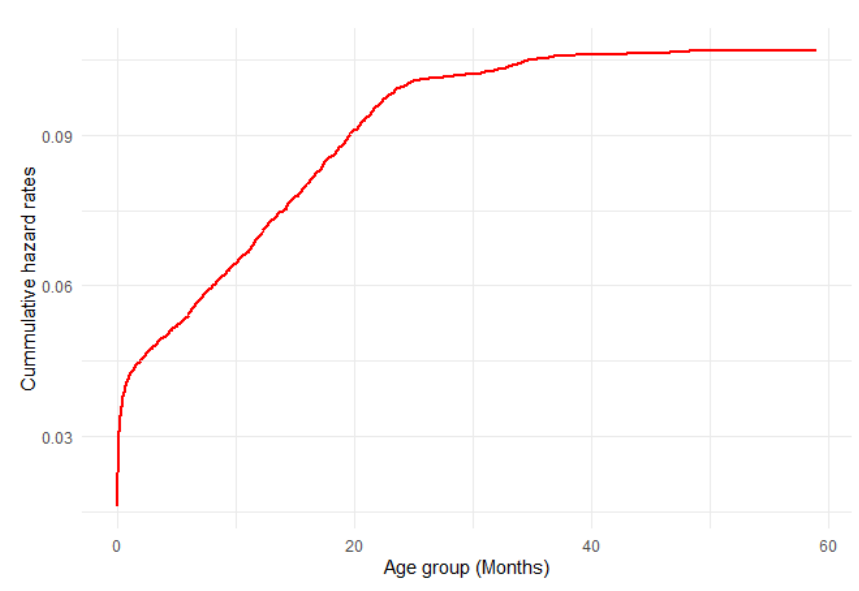

(a)

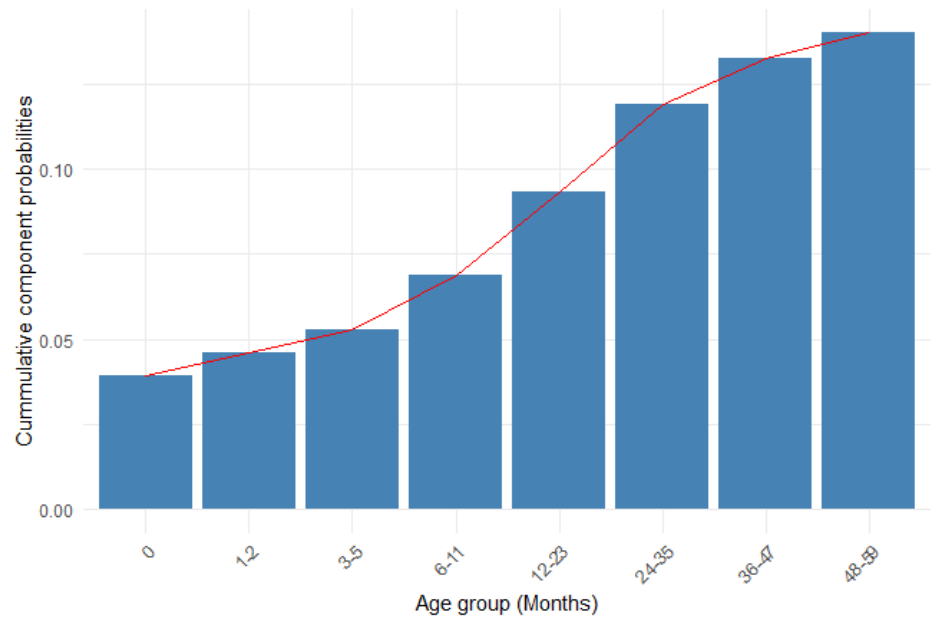

(b)

Figure 2: (a) Nelson Aalen cumulative hazard curve, and (b) cumulative component mortality probability estimate. The highest hazard rate and highest component mortality probability are within the first two months after birth.

study, increased value of model effects implies decreased hazard rate and increased survival probability. Thus, factors with lower effects (coefficients) are considered risk factors to under-five mortality.

\section{Linear Effect}

The linear effect estimates are presented in Table 3, showing the posterior mean, standard deviation (sd), the lower (2.5\%), and upper (97.5\%) quantile of the $95 \%$ credible interval. The reference category for the categorical variables is indicated as "Reference". The non-inclusion of zero in the credible interval was used to determine significance.

The result revealed that female children are significantly less likely to die before their fifth birthday compared with male children, which is the counterpart category. This result is consistent with the Kaplan Meier estimates. Children with mothers 
Table 2: Model adequacy. The Log-normal model with spatial component has the least DIC and WAIC, and thus, it is the best model among the competing models.

\begin{tabular}{l|cc} 
Model & DIC & WAIC \\
\hline Exponential & 23962.29 & 23982.67 \\
Exponential (spatial) & 31477.63 & 22858.4 \\
\hline Gamma & 17268.13 & 17185.25 \\
Gamma (spatial) & 17102.27 & 17003.58 \\
\hline Weibull & 26274.25 & 16825.97 \\
Weibull (spatial) & 26064.64 & 16683.35 \\
\hline Log-normal & 15931.82 & 15825.48 \\
Log-normal (spatial) & $\mathbf{1 5 7 8 4 . 9 8}$ & $\mathbf{1 5 6 7 9 . 9 7}$ \\
\hline Cox & 416677.3 & $4.719455 \mathrm{e}+157$ \\
Cox (spatial) & 126512.6 & $9.277956 \mathrm{e}+62$ \\
\hline
\end{tabular}

who achieved at least a primary level of education are more likely to attain age five compared with children whose mothers did not have any formal education. Moreover, as mothers' education increases, the more likely a child will celebrate the fifth birthday. This is as expected as mothers who achieved a reasonable level of education would have a better knowledge of children's health and nutrition and would likely seek early expert opinion. A similar pattern is found for the household wealth quantile. Children whose household wealth quantile is at least poorer are significantly more likely to attain the fifth birthday compared with the poorest category. As the wealth quantile increases, the more likely a child would survive the fifth birthday.

Children living in a household with improved water and toilet facility are significantly more likely to celebrate the fifth birthday compared with the counterpart, which is unimproved water and toilet facility. It is expected since improved drinking water sources and improved toilet facilities play a significant role in under-five health. The result shows that children living in rural areas are significantly more likely to attain the fifth birthday compared with their counterparts living in the urban settlement. Children whose parents pay attention to at least one mass media means are significantly more likely to attain age five. Children whose parents visit antenatal care more than five times before birth is not significantly different from those whose parents had 2-4 visits before childbirth. Surprisingly, children whose parents do not visit antenatal care are more likely to attain age five compared with the base category. However, for postnatal care utilization, this is not the case. Children whose parent frequently visits postnatal care are significantly more likely to attain age five.

Children not being breastfed are significantly more likely to die before their fifth birthday. Unexpectedly, the result indicated that home-delivered children are less likely to die before age five compared with their counterparts who were delivered in a health facility. Many Nigerian women perceive that childbirth is a normal process and lacks the cultural acceptability of health facility usage and others believe that health facility is only necessary when there are complications in childbirth. Children whose mothers have been working (employed) in the last twelve months are more likely to attain age five compared with children whose mothers have not been working in the last twelve months before the survey. For the type of religion, children whose religion is Catholic, Islam, Traditional, and Others are more likely to attain their fifth birthday compared with the base category, which is other Christians. Children whose birth was multiple are more likely to die before age five. It is 
expected since family resources would be shared among the multiple children. The result indicates that the birth position is not significant.

The result indicates that the mode of delivery does not significantly contribute to the survival of a child. As expected, children who took tetanus injections are less likely to die before age five compared with the children who do not. Surprisingly, children whose household has a bednet are more likely to die before the fifth birthday compared with children whose household does not have a bednet. However, children who sleep under bednets are less likely to die before age five. This result indicates that there is a poor usage of bednets among households with bednets, despite the consistent bednet supply to mitigate the impact of malarial, [29]. Children whose both parents or spouses decide the health care use are more likely to attain the fifth birthday compared with the children whose health care use is only decided by the respondent alone. Moreover, children whose health care use is decided by someone else are not significantly different from the base category. Access to a health care facility does not significantly improve the likelihood a child would survive age five. It could also be attributed to the usage of health care facilities, in that many households do not utilize the healthcare facility even while it is fully accessible.

\section{Spatial Effect}

Figure 3 presents the spatial effect of a child dying before the fifth birthday. In Figure 3a the reddish States represent the states with more prevalence of underfive mortality, while the greenish States are regions with less prevalence. Figure $3 \mathrm{~b}$ presents the probability of elevated risk of a child dying before attaining age five. In general, the northern region of Nigeria is at a higher risk of under-five mortality. In other words, the prevalence of child mortality before the fifth birthday is highly prevalent in the Northern part of Nigeria compared with the southern part of Nigeria. Among the Northern Nigeria states, Kebbi, Kaduna, and Jigawa states have above 0.70 probability of elevated risk of under-five mortality compared with the overall average risk. That is, the prevalence of child mortality in these regions is above $70 \%$ percent higher compared to the country's average prevalence. Invariably, Kebbi, Kaduna, and Jigawa can be tagged child mortality hotspots in Nigeria. These are followed by Adamawa, Gombe, Kanu, Kogi, Nasarawa, Plateau, Sokoto states. However, turning attention to the southern region of Nigeria, Ekiti, Imo, and Osun states have below 0.25 probability of having elevated risk of a child dying before attaining age five compared to the overall average risk. It indicates that the prevalence of under-five mortality in these regions compared to the country's average prevalence is below $25 \%$.

\section{Non-Linear Effect}

Figure 4 presents the nonlinear effects of maternal age and child's birth weight on child mortality before attaining the fifth birthday. In general, the shapes of the effect of maternal age and birth weight on child mortality is an "n" shape. In Figure 4a, the likelihood that a child will attain the fifth birthday increases gradually as the maternal age increases from 15 years and attains a peak at the age of 27 years, and maintains the peak for further 6 years, and then gradually decreases through age 35 to age 49 years. This indicates that there is more prevalence of child mortality 
Table 3: Fixed effects. The non-inclusion of zero in the credible interval was used to decide the significance of a factor. A significantly lower posterior mean indicates a significantly higher risk factor to under-five mortality.

\begin{tabular}{|c|c|c|c|c|}
\hline Variables & Posterior mean & Posterior sd & $2.5 \% \mathrm{Cl}$ & $97.5 \% \mathrm{Cl}$ \\
\hline \multicolumn{5}{|l|}{ Gender } \\
\hline Male & Reference & & & \\
\hline Female & 0.226 & 0.035 & 0.157 & 0.294 \\
\hline \multicolumn{5}{|l|}{ Maternal Education } \\
\hline No formal education & Reference & & & \\
\hline Primary & 0.153 & 0.056 & 0.043 & 0.263 \\
\hline Secondary & 0.209 & 0.054 & 0.103 & 0.315 \\
\hline Higher & 0.253 & 0.091 & 0.074 & 0.432 \\
\hline \multicolumn{5}{|l|}{ Wealth quantile } \\
\hline Poorest & Reference & & & \\
\hline Poorer & 0.106 & 0.049 & 0.009 & 0.203 \\
\hline Middle & 0.177 & 0.056 & 0.067 & 0.287 \\
\hline Rich & 0.277 & 0.066 & 0.147 & 0.407 \\
\hline Richest & 0.409 & 0.082 & 0.249 & 0.569 \\
\hline \multicolumn{5}{|l|}{ Source of water } \\
\hline Unimproved & Reference & & & \\
\hline Improve & 0.078 & 0.037 & 0.006 & 0.151 \\
\hline \multicolumn{5}{|l|}{ Toilet facility } \\
\hline Unimproved & Reference & & & \\
\hline Improve & 0.100 & 0.042 & 0.017 & 0.182 \\
\hline \multicolumn{5}{|l|}{ Residence } \\
\hline Urban & Reference & & & \\
\hline Rural & 0.169 & 0.041 & 0.089 & 0.250 \\
\hline \multicolumn{5}{|l|}{ Mass media usage } \\
\hline No & Reference & & & \\
\hline Yes & 0.077 & 0.039 & 0.000 & 0.155 \\
\hline Frequency of Antenatal care visit & & & & \\
\hline No & 0.474 & 0.064 & 0.348 & 0.600 \\
\hline $2-4$ & Reference & & & \\
\hline $5+$ & 0.071 & 0.044 & -0.015 & 0.156 \\
\hline Frequency of postnatal care visit & & & & \\
\hline No & Reference & & & \\
\hline Yes & 0.246 & 0.065 & 0.118 & 0.374 \\
\hline Child was being breastfed & & & & \\
\hline Yes & Reference & & & \\
\hline No & -3.207 & 0.064 & -3.332 & -3.082 \\
\hline Place of delivery & & & & \\
\hline Health facility & Reference & & & \\
\hline Home & 0.220 & 0.042 & 0.137 & 0.303 \\
\hline Mother has being working in the last & & & & \\
\hline No & Reference & & & \\
\hline Yes & 0.181 & 0.038 & 0.106 & 0.255 \\
\hline Marital status & & & & \\
\hline Never Married & Reference & & & \\
\hline Married & 0.394 & 0.053 & 0.290 & 0.499 \\
\hline Divorced/Spouse Diseased/Separated & 0.388 & 0.116 & 0.160 & 0.617 \\
\hline Religion & & & & \\
\hline Other Christian & Reference & & & \\
\hline Catholic & 0.264 & 0.079 & 0.109 & 0.420 \\
\hline Islam & 0.139 & 0.049 & 0.043 & 0.234 \\
\hline Traditional & 0.156 & 0.312 & -0.444 & 0.778 \\
\hline Others & 0.414 & 0.374 & -0.303 & 1.166 \\
\hline Birth & & & & \\
\hline Single birth & Reference & & & \\
\hline Multiple birth & -0.875 & 0.077 & -1.025 & -0.724 \\
\hline Birth orders & & & & \\
\hline First birth & 0.103 & 0.055 & -0.006 & 0.212 \\
\hline $2-4$ & Reference & & & \\
\hline $5+$ & -0.046 & 0.047 & -0.139 & 0.047 \\
\hline Mode of delivery & & & & \\
\hline Normal & Reference & & & \\
\hline Cesarean & 0.040 & 0.116 & -0.187 & 0.269 \\
\hline Tetanus Injection & & & & \\
\hline No & Reference & & & \\
\hline Yes & 0.531 & 0.043 & 0.447 & 0.615 \\
\hline Household have bednet & & & & \\
\hline No & Reference & & & \\
\hline Yes & -0.178 & 0.044 & -0.264 & -0.093 \\
\hline Children sleeps under bednet & & & & \\
\hline No & Reference & & & \\
\hline Yes & 0.529 & 0.042 & 0.446 & 0.611 \\
\hline Who decide health care use & & & & \\
\hline Respondent alone & Reference & & & \\
\hline Both & 0.160 & 0.056 & 0.051 & 0.269 \\
\hline Spouse & 0.230 & 0.050 & 0.132 & 0.327 \\
\hline Someone else & -0.226 & 0.341 & -0.887 & 0.451 \\
\hline Access to health care & & & & \\
\hline Limited Access & Reference & & & \\
\hline Full Access & -0.038 & 0.292 & -0.603 & 0.543 \\
\hline
\end{tabular}




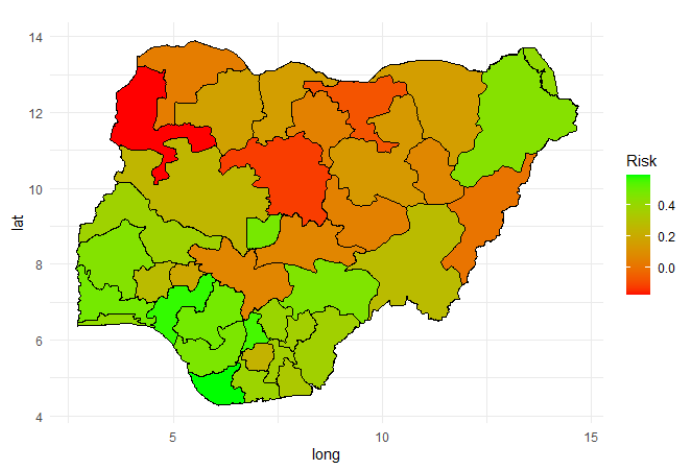

(a)

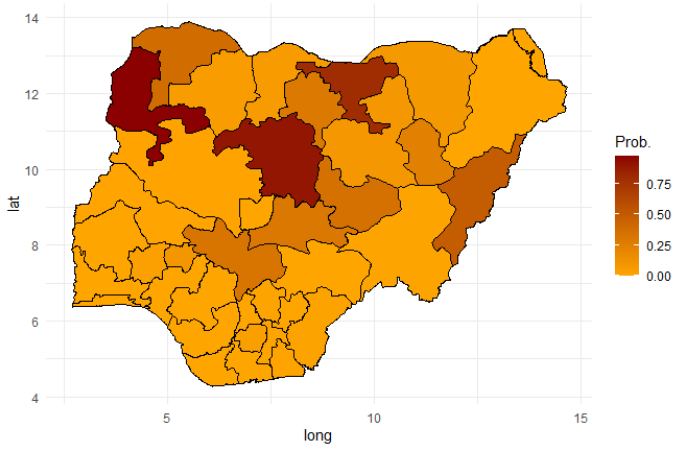

(b)

Figure 3: (a) The spatial effect of state base survival risk, and (b) is the probability of elevated risk of mortality before attaining the fifth birthday. The red states indicate regions with a higher risk of mortality compared to the overall average. States with higher probability indicate regions with a higher probability of under-five death before attaining the fifth birthday.

among young women and older women compared to women within the age range of 24 to 44 years. For younger women, this could be attributed to a lack of experience on a child's nutritional needs or could be attributed to the biological and social mechanism, [30]. Whereas for older women, it could be attributed to the prevalence of complications during childbirth and preterm delivery, [31]

Turning attention to Figure $4 \mathrm{~b}$, the likelihood of a child to attain the fifth birthday gradually increases from a birth weight of $0.5 \mathrm{~kg}$ until it attains a peak at $3 \mathrm{~kg}$ and maintains the same effect for an additional $0.5 \mathrm{~kg}$. The likelihood gradually decreases until a birth weight of $6 \mathrm{~kg}$. In other words, the prevalence of child mortality is high among birth weight less than $2.5 \mathrm{~kg}$ and those higher than $4.5 \mathrm{~kg}$. It could be attributed to the prevalence of subnormal growth and neurodevelopmental complications among children with low birth weight, [32]. 


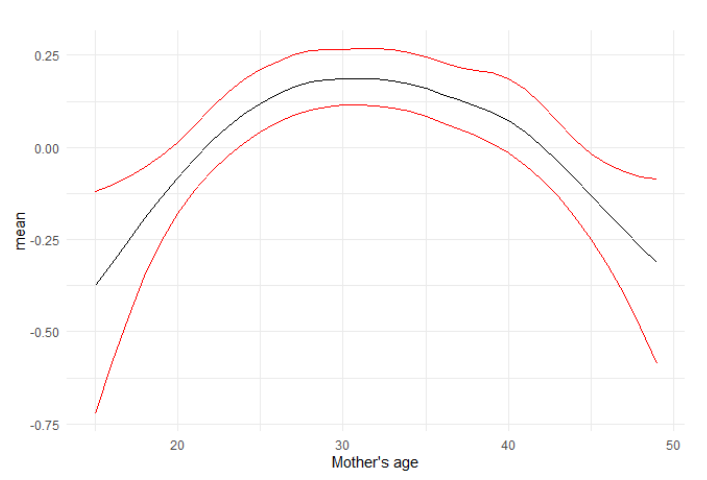

(a)

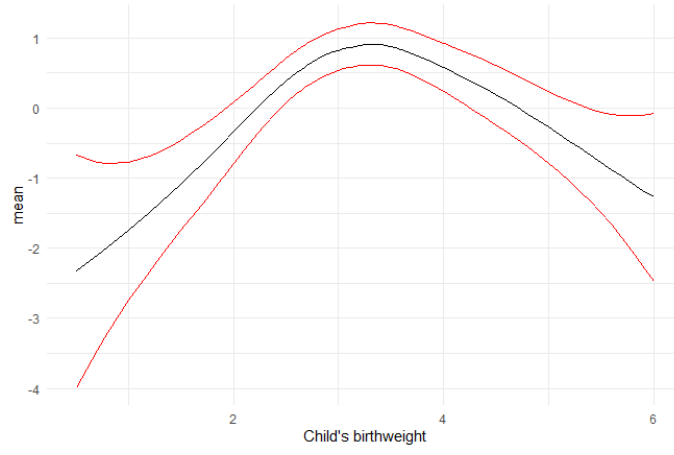

(b)

Figure 4: (a) The effect of maternal age (years), and (b) child's birth weight (Kg) to under-five mortality before attaining the fifth birthday. The risk is higher at the beginning and end of maternal age, and birth weight below $2.5 \mathrm{~kg}$ and above $4.5 \mathrm{~kg}$.

\section{Discussion}

This study considered the time to death outcomes of under-five children in Nigeria. A Bayesian hierarchical hazard modeling framework was adopted. Five models, including Exponential, Gamma, Weibull, Log-normal, and Cox hazard models were fitted, with and without the inclusion of geographically varying effects. Specifically, this study formulated these hazard models in a latent Gaussian framework capable to be analyzed using INLA. The adequacy of the models was checked using the WAIC and DIC criteria. Among all models, the log-normal model stood out as the most suitable model to explain the variations in the time to death outcomes variable. The nonparametric Kaplan-Meier survival curve, Nelson-Aalen cumulative hazard curve, and the cumulative component mortality probability were also estimated to understand mortality risk through the five years before the fifth birthday. Findings show that Gender, Maternal Education, household wealth status, source of water and toilet facility, residence, mass media, frequency of antenatal and postnatal visits, marital status, place of delivery, multiple births, who decide healthcare use, use of bednet are significant risk factors of child mortality. Findings also indicate disparity in geographical regions, across mother's age, and birth weight. 
Findings show that the survival probability of under-five children has the highest fall within the first two months after birth. That is, children within the first two months of birth have the highest expected risk of death. This finding advocate that mortality at this early age significantly hikes the under-five mortality rate in Nigeria. Hence, this study suggests that intervention programs set to mitigate mortality should give priority to children within the first two months as this could effectively scale down the overall under-five mortality index in Nigeria. Findings indicate that male children have an elevated risk of dying before their fifth birthday compared with female children in the same age group. This finding is consistent with biological results that male children are biologically weaker and more susceptible to diseases compared with female children, [33, 34]. Findings showed that attainment of higher maternal education increases the survival of children. Improving mothers' maternal education from no education to primary education has the largest contribution to mitigating mortality rate compared with the improvement from primary to secondary or secondary to higher. Thus, mass mothers' participation in primary maternal education could speed the achievement of the Sustainable Development Goals. This finding is expected as educated parents are more likely to have a positive attitude towards parenting, often seek expert advice, and involve in a child's physical and mental growth, $[35,36]$. Thus, this finding suggests that maternal education is among the key important markers to mitigate the high mortality rate in Nigeria.

This study identified a discrepancy in the risk of mortality among households' wealth status. That is, the risk of mortality is most severe among the poorest wealth quantile, and the risk consistently decreases with an increase in wealth quantile. The most prominent increase in under-five survival was found for a one-step wealth quantile increase from the rich to the richest. However, for intervention, improving household wealth quantile from rich to richest seems impractical, rather programs could focus on the improvement from poorest to poorer quantile by providing basic living conditions, such as access to good shelter, road, and electricity being that the improvement from poorest to poorer wealth quantile is the second most prominent increase in under-five survival probability. This finding is consistent with existing studies, [37, 38]. Wealth inequalities, non-diversification of the economy, unemployment, and low education financing in Nigeria are the major key factors contributing to increasing poverty, [39], and consequently, increase risk of child mortality in Nigeria. This finding identifies poverty as a leading cause of mortality. Poverty alleviation programs to improve the livelihoods of households are one of the main remedies to child mortality in Nigeria.

Findings also indicated that access to an improved water source and toilet facility lowers the risk of mortality. However, the improvement is more prominent for the toilet facilities. Thus, policies to safely managed improved household water sources and toilet facilities are required to mitigate child mortality, [40]. Surprisingly, the frequency of antenatal care visits does not improve the survival probabilities of children. However, the survival probability increases for postnatal visits. It could be associated with late first-time antenatal utilization or irregular visitation. Women tend to wait towards the delivery period or complications during gestation before utilizing antenatal care services. Thus, educating mothers on the importance of 
early antenatal utilization could scale down the under-five mortality rate. Similarly, home delivery in Nigeria significantly improves the survival probabilities of a child to celebrate the fifth birthday. This finding is contrary to the common rationale that babies delivered in a health facility are more likely to attain their fifth birthday since medical experts are readily available to give the first medical support and advice. According to [6], the prevalence of non-utilization of health facilities for childbirth is about $62 \%$ of the total birth in Nigeria. It could be associated to poor quality of health facility, caring, cost and cultural believes that lacks the acceptability of health care services, and the believes that healthcare facility in only necessary during birth complications, [41]. Thus, intervention programs that give financial support and prenatal education are needed to encourage the frequent use of health facilities but not only when there are complications during childbirth.

Findings indicate that maternal occupation since a year before the survey significantly lowers the risk of child mortality. This finding is in line with a previous study, [42]. However, the reverse may be the case if only children under breastfeeding were considered, [43]. Findings indicate that married couples increase the survival probabilities of children to attain their fifth birthday. Nonetheless, divorced, deceased spouses or separated parents also increase survival compared to never-married parents. It could be linked to the elevated likelihood of children of unmarried parents to surfer the consequence of limited resources, poverty, and poor schooling, [44, 45]. This study also found that multiple births significantly increases the risk of underfive mortality compared with singleton. It could be associated with the consequence of shared limited family resources among the children. This finding suggests that multiple births jeopardize the survival of under-five children. Hence, children of multiple births should be given special treatment in addition to positive involvement in parental support. Findings also indicate that immunization with Tetanus strongly increases the survival of children. This finding is in line with the result of a previous study, [46]. Thus, immunization with Tetanus and its benefits on the under-five survival should be well publicized and target parents with low maternal education attainment. This study found that household with bednets does not lower the risk of child mortality. However, the usage of insecticide-treated bednets among children significantly lowers the risk of mortality in children. The magnitude of the effect of bednet use in the improvement of child survival is equivalent to the magnitude of immunization with Tetanus. This finding suggests that the non-usage of insecticide-treated bednet increases the risk of mortality, and it should be considered as a significant risk factor. Thus, intervention programs that donate and distribute insecticide-treated bednets to households in Nigeria should take it as an additional duty to educate parents on the importance of the use of bednets on the survival of children. This finding is consistent with a previous study in Ghana, [47]. This study found that both parent and husband's decision on the mother's health use significantly improves child survival compared to mother decision alone. Moreover, a someone-else decision on the mother's use of health services increases the risk of mortality. This finding suggests that decisions on mother's health use outside the parents could increase the risk of mortality. Hence, at least spouses (fathers) should be involved in the decision-making on health use. The role of the father to a child's health plays a crucial part in child survival, as studies have shown that the father's involvement is related to positive child health outcomes, [48]. 
This study also substantiates the effect of maternal age and birth weight on child mortality. Findings indicate that the risk of mortality among under-age five children increases for maternal age below 24 and above 44 years. This finding suggests that maternity age in the mentioned disjoint age intervals are risk factors to child mortality. Similarly, under age five mortality is high at lowest birth weights, improves within the most frequent average birth weights, and then picks again at highest birth weights. Specifically, it was found that birth weight less than $2.5 \mathrm{~kg}$ and above $4.5 \mathrm{~kg}$ elevates the risk of underage five mortality. These findings are consistent with previous studies, [49, 50].

This study identified significant spatial heterogeneity in the risk of under-five mortality before attaining the fifth birthday. Findings show a clear divide between North and South Nigeria, placing the Northern states at higher risk regions compared with the South. Specifically, among the Northern states in Nigeria, this study identified Kebbi, Kaduna, and Jigawa as the hotspot of child mortality in Nigeria. Additionally, Adamawa, Gombe, Kanu, Kogi, Nasarawa, Plateau, Sokoto states were also identified as high-risk regions. This finding is consistent with previous studies, [12].

\section{Conclusion}

This study quantified the risk of child mortality over the first five years in the form of probabilities before attaining the fifth birthday and also identified the risk factors elevating the under-five mortality rate in Nigeria. These findings call for action on the identified risk factors to mitigate the under-five mortality rate in Nigeria. This study accentuates the need for special attention for the first two months after childbirth as it is the age group with the highest expected mortality. A practicable way to minimize death in the early life of children is to improve maternal health care service, promote maternal education, encourage delivery in healthcare facilities, positive parental attitude to support multiple births, poverty alleviation programs for the less privileged, and a prioritized intervention to Northern Nigeria. The identified hotspot could be used for policy-making and as a guide towards the distribution of intervention resources. Additionally, it could serve for the construction of a localized framework for intervention program evaluation. The maps produced in this study could be placed beside another map, such as poverty, unemployment rate, or insurgence maps to identify the high burden regions across the country.

The prime limitation of this study is the noninclusion of regional factors that directly influence the survival probabilities of children. These factors include food security and child nutritional intake. The adopted model could suffer from the omission of diseased children from the survey and the missed information of the exact day of death, and consequently affect the point survival probability estimates. There is nonavailability of data on the cause of child death to account for the risk of unavoidable deaths and its impact on research results.

\section{List of Abbreviations}

DIC: Deviance Information criterion. EA: Enumeration Areas. NDHS: Nigeria Demographic and Health Survey. NPC: National Population Commission. R-INLA: R-Integrated Nested Laplace Approximation. UNICEF: United Nations Children's 
Fund. WAIC: Watanabe-Akaike Information Criterion . WHO: World Health Organization.

\section{Acknowledgements}

Osafu Augustine Egbon and Mariella Ananias Bogoni acknowledge the support from the Brazilian institution CAPES. Francisco Louzada acknowledges the support from the Brazilian institution CNPq and FAPESP. The authors would like to acknowledge the Demographic and Health Survey program for granting access to the data used in the study.

\section{Declaration \\ Funding}

No funding

\section{Availability of data and materials}

The Nigeria Demographic and health survey data used in this study are available and accessible on the DHS program website (http://dhsprogram.com).

Ethics approval and consent to participate

All procedures performed in this study involving human participants were in accordance with the ethical standards of the institutional and/or national research committee and with the 1964 Helsinki declaration and its later amendments or comparable ethical standards.

Competing interests

The authors declare that they have no competing interests.

Consent for publication

Not applicable.

\section{Authors' contributions}

OAE conceived the study idea, BTB and MAB sourced the data, OAE and MAB performed the computation, FL and $B T B$ validated the model. All authors participated in the manuscript writing, read and accepted the final manuscript revision.

\section{Author details}

${ }^{1}$ Institute of Mathematical and Computer Sciences, University of São Paulo, São Carlos, Brazil. ${ }^{2}$ Department of Statistics, Universidade Federal de São Carlos, São Carlos, Brazil. ${ }^{3}$ Department of Mathematics and Statistics, Kampala International University, Kampala, Uganda.

\section{References}

1. UNICEF: Levels and trends in child mortality. Technical report, eSocialSciences (2020)

2. WHO, et al.: Children: Improving survival and well-being (2020)

3. Unicef: UNICEF Data: Monitoring the situation of children and women. https://data.unicef .org/country/nga/ (2020)

4. Unicef, et al.: Nigeria multiple indicator cluster survey 2016-2017. Technical report, NBS, UNICEF (2018)

5. Adedini, S.A., Odimegwu, C., Imasiku, E.N., Ononokpono, D.N.: Ethnic differentials in under-five mortality in nigeria. Ethnicity \& health 20(2), 145-162 (2015)

6. Adedokun, S.T., Uthman, O.A.: Women who have not utilized health service for delivery in nigeria: who are they and where do they live? BMC Pregnancy and Childbirth 19(1), 1-14 (2019)

7. PMNCH: Women's children's and adolescents' health (2021)

8. Olopha, P.O., Fasoranbaku, A.O., Gayawan, E.: Spatial pattern and determinants of sufficient knowledge of mother to child transmission of hiv and its prevention among nigerian women. Plos one 16(6), 0253705 (2021)

9. Angela, C., Uju, O.: Determinants of under-five mortality in nigeria: an application of cox proportional hazard and cox frailty models. IOSR J Math Ver I [Internet] 11(4), 2278-5728 (2015)

10. Adeyinka, D.A., Muhajarine, N., Petrucka, P., Isaac, E.W.: Inequities in child survival in nigerian communities during the sustainable development goal era: insights from analysis of 2016/2017 multiple indicator cluster survey. BMC public health 20(1), 1-18 (2020)

11. Ayoade, M.A.: Spatio-temporal patterns of under 5 mortality in nigeria. SSM-population health 6, 116 (2018)

12. Fagbamigbe, A.F., Nnanatu, C.C.: Modelling the spatial distribution and the factors associated with under-five mortality in nigeria. Spatial Demography, 1-28 (2021)

13. DHS: Guide to DHS statistics DHS-7. https://dhsprogram.com/data/Guide-to-DHS-Statistics/Early_Childhood_Mortality.htm (2021)

14. Sinha, S., Aggarwal, A.R., Osmond, C., Fall, C.H., Bhargava, S.K., Sachdev, H.S.: Maternal age at childbirth and perinatal and under-five mortality in a prospective birth cohort from delhi. Indian pediatrics 53(10), 871-877 (2016)

15. Royston, P., Altman, D.G., Sauerbrei, W.: Dichotomizing continuous predictors in multiple regression: a bad idea. Statistics in medicine 25(1), 127-141 (2006)

16. MacCallum, R.C., Zhang, S., Preacher, K.J., Rucker, D.D.: On the practice of dichotomization of quantitative variables. Psychological methods 7(1), 19 (2002)

17. WHO/UNICEF: Joint Monitoring Programme for Water Supply and Sanitation: Progress on sanitation and drinking-water: 2010 update. World Health Organization (2010)

18. Cox, D.R.: Regression models and life-tables. Journal of the Royal Statistical Society. Series B (Methodological) 34(2), 187-220 (1972) 
19. Lindgren, F., Rue, H.: On the second-order random walk model for irregular locations. Scandinavian journal of statistics 35(4), 691-700 (2008)

20. Martino, S., Riebler, A.: Integrated nested laplace approximations (inla). Wiley StatsRef: Statistics Reference Online, 1-19 (2014)

21. R Core Team: R: A Language and Environment for Statistical Computing. R Foundation for Statistical Computing, Vienna, Austria (2019). R Foundation for Statistical Computing. https://www.R-project.org/

22. Ruiz-Cárdenas, R., Krainski, E.T., Rue, H.: Direct fitting of dynamic models using integrated nested laplace approximations-inla. Computational Statistics \& Data Analysis 56(6), 1808-1828 (2012)

23. Martins, T.G., Simpson, D., Lindgren, F., Rue, H.: Bayesian computing with inla: new features. Computational Statistics \& Data Analysis 67, 68-83 (2013)

24. Blangiardo, M., Cameletti, M., Baio, G., Rue, H.: Spatial and spatio-temporal models with r-inla. Spatial and spatio-temporal epidemiology 4, 33-49 (2013)

25. Palmi-Perales, F., Gomez-Rubio, V., Martinez-Beneito, M.A.: Bayesian multivariate spatial models for lattice data with inla. arXiv preprint arXiv:1909.10804 (2019)

26. Egbon, O., Somo-Aina, O., Gayawan, E.: Spatial weighted analysis of malnutrition among children in nigeria: A bayesian approach. Statistics in Biosciences 35(4), 1-29 (2021). doi:10.1007/s12561-021-09303-9

27. United Nations: Mortality estimates from major sample surveys: towards the design of a database for the monitoring of mortality levels and trends. United Nations, Department of Economic and Social Affairs (2011)

28. Egbon, O.A., Somo-Aina, O., Gayawan, E.: Spatial weighted analysis of malnutrition among children in nigeria: A bayesian approach. Statistics in Biosciences, 1-29 (2021)

29. Russell, C.L., Sallau, A., Emukah, E., Graves, P.M., Noland, G.S., Ngondi, J.M., Ozaki, M., Nwankwo, L., Miri, E., McFarland, D.A., et al.: Determinants of bed net use in southeast nigeria following mass distribution of Ilins: implications for social behavior change interventions. PLoS One 10(10), 0139447 (2015)

30. Finlay, J.E., Özaltin, E., Canning, D.: The association of maternal age with infant mortality, child anthropometric failure, diarrhoea and anaemia for first births: evidence from 55 low-and middle-income countries. BMJ open 1(2), 000226 (2011)

31. Cavazos-Rehg, P.A., Krauss, M.J., Spitznagel, E.L., Bommarito, K., Madden, T., Olsen, M.A., Subramaniam, H., Peipert, J.F., Bierut, L.J.: Maternal age and risk of labor and delivery complications. Maternal and child health journal 19(6), 1202-1211 (2015)

32. Hack, M., Klein, N.K., Taylor, H.G.: Long-term developmental outcomes of low birth weight infants. The future of children, 176-196 (1995)

33. Costa, J.C., da Silva, I.C.M., Victora, C.G.: Gender bias in under-five mortality in low/middle-income countries BMJ global health 2(2), 000350 (2017)

34. Pongou, R.: Why is infant mortality higher in boys than in girls? a new hypothesis based on preconception environment and evidence from a large sample of twins. Demography 50(2), 421-444 (2013)

35. Andriano, L., Monden, C.W.: The causal effect of maternal education on child mortality: Evidence from a quasi-experiment in malawi and uganda. Demography 56(5), 1765-1790 (2019)

36. Cleland, J.G., Van Ginneken, J.K.: Maternal education and child survival in developing countries: the search for pathways of influence. Social science \& medicine 27(12), 1357-1368 (1988)

37. Chao, F., You, D., Pedersen, J., Hug, L., Alkema, L.: National and regional under- 5 mortality rate by economic status for low-income and middle-income countries: a systematic assessment. The Lancet Global Health 6(5), 535-547 (2018)

38. Cha, S., Jin, Y.: Have inequalities in all-cause and cause-specific child mortality between countries declined across the world? International journal for equity in health 19(1), 1-13 (2020)

39. Ucha, C.: Poverty in nigeria: Some dimensions and contributing factors. Global Majority E-Journal 1(1), 46-56 (2010)

40. Ezeh, O.K., Agho, K.E., Dibley, M.J., Hall, J., Page, A.N.: The impact of water and sanitation on childhood mortality in nigeria: evidence from demographic and health surveys, 2003-2013. International journal of environmental research and public health 11(9), 9256-9272 (2014)

41. Konje, E.T., Hatfield, J., Kuhn, S., Sauve, R.S., Magoma, M., Dewey, D.: Is it home delivery or health facility? community perceptions on place of childbirth in rural northwest tanzania using a qualitative approach. BMC pregnancy and childbirth 20(1), 1-11 (2020)

42. Akinyemi, J.O., Solanke, B.L., Odimegwu, C.O.: Maternal employment and child survival during the era of sustainable development goals: Insights from proportional hazards modelling of nigeria birth history data. Annals of global health 84(1), 15 (2018)

43. Bankole, A.: Maternal employment and infant mortality: an examination of the role of breastfeeding as an intermediate factor. Biology and society: the journal of the Eugenics Society 6(1), 19-26 (1989)

44. Clark, C., Hamplová, D.: The impact of mother's marital status on child mortality in Sub-Saharan Africa: An analysis of birth and marital histories. Canada: Canada Research Chair in Youth, Gender and Global Health (2011)

45. McLanahan, S., Sandefur, G.: Growing up with a Single Parent. What Hurts, What Helps. ERIC, ??? (1994)

46. Blencowe, H., Lawn, J., Vandelaer, J., Roper, M., Cousens, S.: Tetanus toxoid immunization to reduce mortality from neonatal tetanus. International journal of epidemiology 39(suppl_1), 102-109 (2010)

47. Afoakwah, C., Nunoo, J., Andoh, F.K.: Effect of insecticide-treated bed net usage on under-five mortality in northern ghana. Malaria journal 14(1), 1-6 (2015)

48. Nair, S., Chandramohan, S., Sundaravathanam, N., Rajasekaran, A.B., Sekhar, R.: Father involvement in early childhood care: insights from a mel system in a behavior change intervention among rural indian parents. Frontiers in Public Health 8, 516 (2020)

49. Kim, Y.-N., Choi, D.-W., Kim, D.S., Park, E.-C., Kwon, J.-Y.: Maternal age and risk of early neonatal mortality: a national cohort study. Scientific reports 11(1), 1-9 (2021)

50. Wilcox, A.J., Russell, I.T.: Birthweight and perinatal mortality: Ii. on weight-specific mortality. International journal of epidemiology 12(3), 319-325 (1983) 